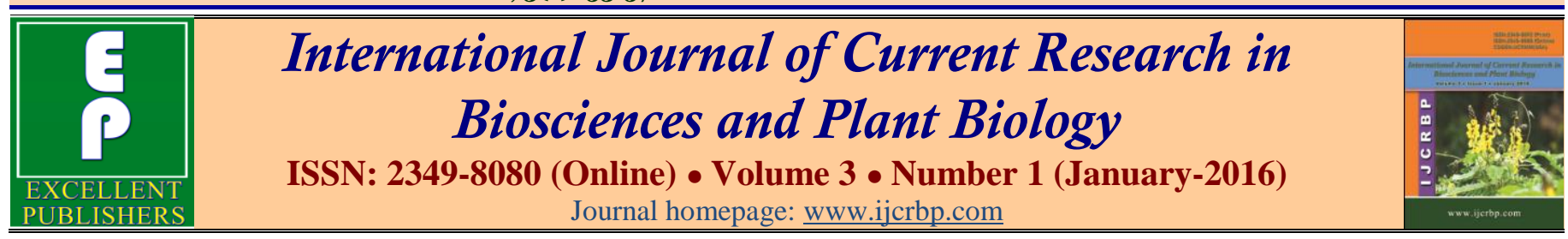

\title{
Theoretical Evaluation of Corrosion Inhibition Performance of Some Benzenthiol Derivatives for Copper in Acid Media
}

\author{
M. Thyab K. Al-Baalawi ${ }^{1 *}$ and M. K. Muhammed ${ }^{2}$ \\ ${ }^{1}$ Marine Chemistry Department, Marine Science Center, University of Basra, Iraq \\ ${ }^{2}$ Ministry of Environment, Department of Protection and Improvement of Environment, Northern Region, Kirkuk, Iraq \\ *Corresponding author.
}

\section{Abstract}

The adsorption mechanism and inhibition performance of two benzenthiol derivatives benzenthiol (BT) and 4-mehtyl-benzenthiol (4-M) and 4-isopropylbenzenthiol (4Iso) were investigated as corrosion inhibitors using semiempirical methods (AM1 and PM3) and ab-nitio (HF). The result showed that the theoretically calculated order was found to be in close agreement with the experimental order. The calculated quantum chemical parameters correlated to the inhibition efficiency are HOMO energy level (highest occupied molecular orbital energy), LUMO energy level (lowest unoccupied molecular orbital energy), the energy gap $(\Delta \mathrm{E})$, hardness $(\eta)$, electrophilicity $(\omega)$.

\section{Article Info}

Accepted: 14 December 2015

Available Online: 06 January 2016

Keywords

Adsorption

Benzenthiol

Copper

HOMO energy

Inhibitor group

LUMO energy

\section{Introduction}

Copper is one of the most important material used widely in different industries, especially in central heating installations, oil refiners, energetic and marine environment because of its excellent conductivity, good mechanical workability and relatively low cost and reactivity. It is relatively a noble metal; however, it is susceptible to corrosion by acids and strong alkaline solutions. The use of inhibitors is one of the most practical methods for protecting metals or alloys from corrosion. Inhibitors are chemicals that often work by adsorbing themselves on the metallic surface by forming a film (Noor, 2008; Ebenso et al., 2008; Oguzie, 2006). Organic compounds containing polar groups including nitrogen, sulfur, and oxygen (Otmacic et al., 2004; Ma et al., 2002; Wang et al., 2004; Kendig and Jeanjaquet, 2002; Ma et al., 2003), and heterocyclic compounds with polar functional groups and conjugated double bonds (Elmorsi and Hassanein, 1999;
Al-Kharafi, 1988; Scendo et al., 2003) have been reported to inhibit copper corrosion. The inhibiting action of these organic compounds is usually attributed to their interactions with the copper surface via their adsorption. Even though many synthetic organic compounds showed good anticorrosive activity, most of them are highly toxic to both human beings and the environment. Presently, many researchers have reoriented their studies to the use of naturally occurring substances. Naturally occurring substances are cheap and renewable, biodegradable and do not contain heavy metals or other toxic chemicals and are therefore eco-friendly and hence ecologically acceptable. Most efficient inhibitors are organic compounds containing electronegative functional groups and $\pi$-electrons in triple or conjugated double bonds. The remarkable inhibitory effect is reinforced by the presence of heteroatom such as sulphur, nitrogen and oxygen in the ring which facilitates its adsorption on the metal surface following the sequence $\mathrm{O}<\mathrm{N}<\mathrm{S}$ (Obot et al., 2009). 
Researchers concluded that the adsorption on the metal surface depends mainly on the physicochemical properties of the inhibitor group, such as the functional group, molecular electronic structure, electronic density at the donor atom, $\pi$ orbital character and the molecular size (Obot and Obi-Egbedi, 2008a; Obot and ObiEgbedi, 2008b; Ju et al., 2008; Quraishi et al., 1997). The reaction mechanism includes the transfer of one pair of electrons from the organic compound, and the formation of the coordinate covalent bond with metal or an alloy (Growcock, 1989). Under such conditions, for a given metal, the effiency of the inhibitor depends on the stability of the formed chelate and the inhibitor molecule should have centers, which are capable of forming bonds with metal surface via the electron transfer. In this way the metal acts as an electrophile, where as the inhibitor molecule acts as a Lewis base, whose nucleophile centers are normally hetero atoms with free electron pairs which are readily available for the sharing to form a bond. Recently, theoretical prediction of the efficiency of corrosion inhibitors has become very popular in parallel with the progress in computational hardware and the development of efficient algorithms which assisted the routine development of molecular quantum mechanical calculations (Domenicano and Hargittai, 1992).

The semiempirical and ab-nitio methods of calculation have been used in most successfully completed studies in which the calculated quantum chemical properties are used enables the correlation of the obtained data with inhibitor efficiency (Bereket et al., 2001; Lukovits et al., 2001). The electronic properties of the inhibitors, the effect of the highest occupied molecular orbital (HOMO) and the lowest unoccupied molecular orbital (LUMO) energies, the difference between highest occupied molecular orbital and lowest unoccupied molecular orbital energies $\left(\mathrm{E}_{\mathrm{LUMO}}-\mathrm{E}_{\mathrm{HOMO}}\right)$, charge on the reactive center had to be investigated to achieve the appropriate correlation (Quraishi et al., 1996; Al-Mayouf et al., 1998). Benzothiazoles have been used as corrosion inhibitors for copper (Sankarapapavinasam and Ahmed, 1992). Thiazole derivatives are proved to be effective in protecting copper corrosion in acidic sulfate containing medium.

\section{Materials and methods}

\section{Method of calculations}

Theoretical calculations were carried out by using semiemperical (AM1, PM3) and ab-nitio (HF) in Gamess program attached with BiochemOffice-2008. The experimental data taken from the publication literature (Elmorsi and Hassanein, 1999). Fig. 1 shown below is the optimized structure of benzenthiol and derivatives.

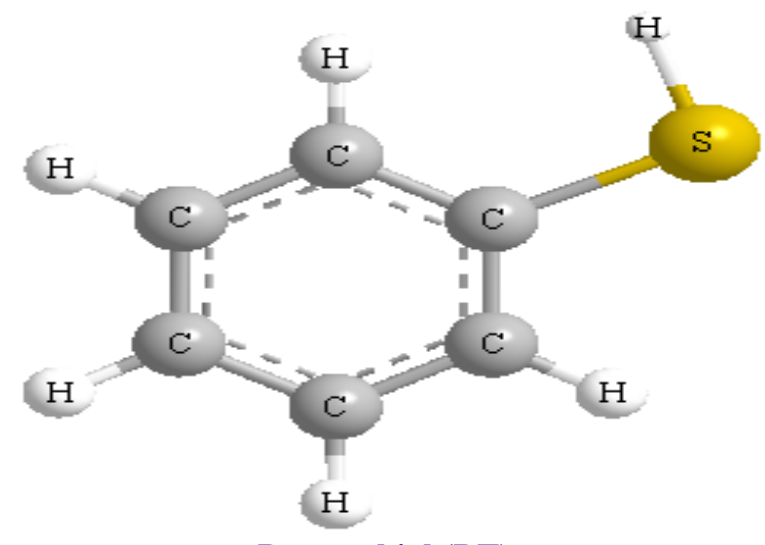

Benzenthiol (BT)

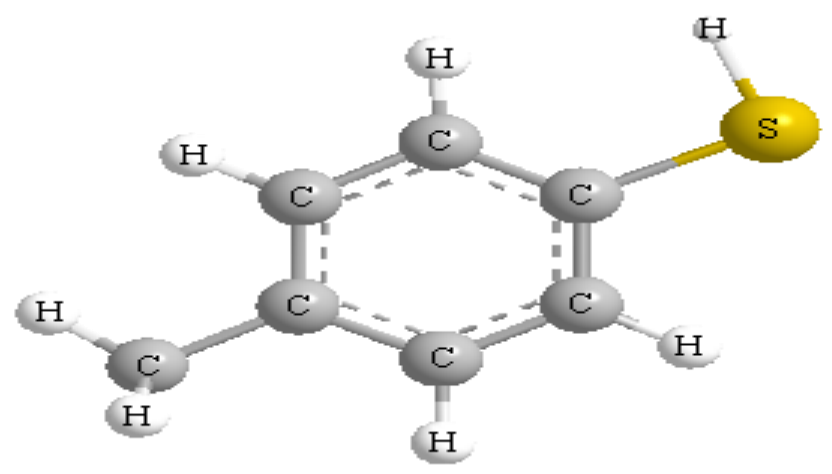

4-mehtyl-benzenthiol (4-M)

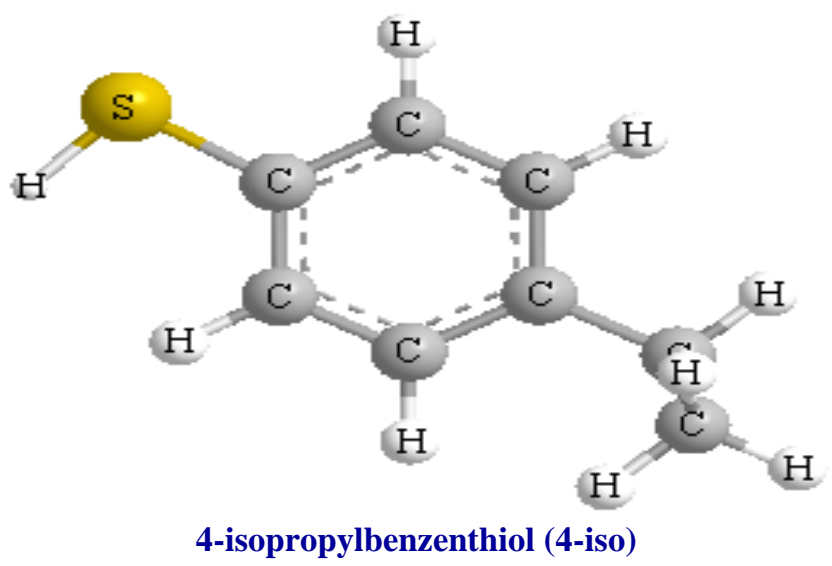

Fig. 1: Optimized structure of benzenthiol derivatives

\section{Results and discussion}

Relationships between inhibition efficiency and the global quantum chemical parameters The inhibition efficiency of the compounds depends on many major factors such as the number of adsorption active centers in the molecule and their HOMO and LUMO energy level, 
the energy gaps $\Delta \mathrm{E}$, Hardness, electro potential chemistry and Global electrophilicity. The substitute effect on the copper inhibition of the benzenthiol compounds. The algebraic values of this quantity are given in Tables 1, 2 and 3 and the experimental data are given in Table 4.

Table 1. Physical parameter by using HF method for inhibitor corrosion.

\begin{tabular}{ccccccc}
\hline Comp. & HOMO $_{\text {energy }}$ & LUMO $_{\text {energy }}$ & $\Delta \mathbf{E}$ & $\boldsymbol{\mu}$ & $\boldsymbol{\eta}$ & $\omega$ \\
\hline 4-iso & -0.2939 & 0.1339 & 0.4278 & -0.08 & 0.2139 & 0.01496 \\
4-M & -0.3035 & 0.1296 & 0.4331 & -0.08695 & 0.21655 & 0.017456 \\
BT & -0.3132 & 0.1335 & 0.4467 & -0.08985 & 0.22335 & 0.018073 \\
\hline
\end{tabular}

Table 2. Physical parameter by using PM3 method for inhibitor corrosion.

\begin{tabular}{ccccccc}
\hline Comp. & HOMO $_{\text {energy }}$ & LUMO $_{\text {energy }}$ & $\Delta \mathbf{E}$ & $\boldsymbol{\mu}$ & $\boldsymbol{\eta}$ & $\omega$ \\
\hline 4-iso & -0.3151 & -0.0009 & 0.3142 & -0.0627 & -0.158 & 0.079453 \\
4-M & -0.3186 & -0.0025 & 0.3161 & -0.16055 & -0.15805 & 0.081545 \\
BT & -0.3186 & -0.0006 & 0.318 & -0.3554 & -0.1596 & 0.091756 \\
\hline
\end{tabular}

Table 3. Physical parameter by using AM1 method for inhibitor corrosion.

\begin{tabular}{ccccccc}
\hline Comp. & HOMO $_{\text {energy }}$ & LUMO $_{\text {energy }}$ & $\Delta \mathbf{E}$ & $\boldsymbol{\mu}$ & $\boldsymbol{\eta}$ & $\omega$ \\
\hline 4-iso & -0.311 & 0.0062 & -0.3172 & -0.1524 & 0.1586 & 0.073221 \\
4-M & -0.3131 & 0.0074 & -0.3205 & -0.15285 & 0.16025 & 0.072896 \\
BT & -0.3119 & 0.0087 & -0.3206 & -0.1516 & 0.1603 & 0.071686 \\
\hline
\end{tabular}

Table 4. Experimental data for inhibition efficiency of benzenthiol derivative.

\begin{tabular}{cccc}
\hline Compound & BT & 4-M & 4-iso \\
\hline IE\% & 74.39 & 82.53 & 86.47 \\
\hline
\end{tabular}

HOMO is the high orbital molecular occupied measures the tendency toward the donation of electron by amolecule (Obot and Obi-Egbedi, 2008b). Therefore higher values of HOMO energy indicate better tendency toward the donation of electron, enhancing the adsorption of the inhibitor on copper and therefore better inhibition efficiency. LUMO energy is the ability of the molecule to accept electron. The binding ability of the inhibitor to the copper surface increase with increasing of the HOMO (Quraishi et al., 1997) but not found relationship between the LUMO and inhibition efficiency, that indicate don't transfer the electron from metal to inhibitors compounds.

The energy gap $\Delta \mathrm{E}\left(\Delta \mathrm{E}=\mathrm{LUMO}_{\text {energy }}-\mathrm{HOMO}_{\text {energy }}\right)$ is an important parameter as a function of reactivity of the inhibitor molecule towards the adsorption on the metallic surface. As $\Delta \mathrm{E}$ decreases the reactivity of the molecule increases leading to increase in the \%IE of the molecule.
Lower values of the energy difference will render good inhibition efficiency, because the energy to remove an electron from the last occupied orbital will be low (ObiEgbedi et al., 2011). A molecule with a low energy gap is more polarizable and is generally associated with the high chemical activity and low kinetic stability and is termed soft molecule (Obot et al., 2009). The results as indicated in Table 1 shows that inhibitor 4-iso has the lowest energy gap, this means that the molecule could have better performance as corrosion inhibitor.

Hardness is important properties to measure the molecular stability and reactivity. It is apparent that the chemical hardness fundamentally signifies the resistance towards the deformation or polarization of the electron cloud of the atoms, ions or molecules under small perturbation of chemical reaction. A hard molecule has a large energy gap (Antonijevic and Petrovic, 2008). The hardness was calculated by using following low:
$H \operatorname{ardness}(\eta)=\frac{L U M O_{\text {ENERGY }}-H O M O_{\text {ENERGY }}}{2}$ 
In our present study 4-iso with low hardness value. compared with other compound have a low energy gap. Normally, the inhibitor with the least value of global hardness is expected to have the highest inhibition efficiency (El Ashry et al., 2006).

Calculated the electro potential chemistry by using following low:

Electro Potential Chemistry

$(\mu)=\frac{L U M O_{E N E R G Y}+H O M O_{E N E R G Y}}{2} \cdots \ldots$.

Furthermore calculated Global Electrophilicty Index by using Electro potential chemistry and Hardness in fowling low:

Global Elactrophilicity Index

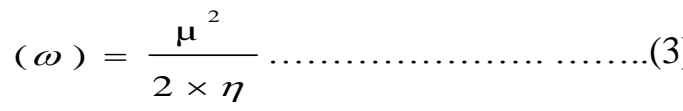

The electrophilicity index $\omega$, shows the ability of the inhibitor molecules to accept electrons. It is a measure of the stabilization in energy after a system accepts additional amount of electron charge from the environment. The Tables 1, 2 and 3 shows the order of Electrophilicity as BT > 4-m > 4-iso.

\section{Conclusion}

It has been noticed that the quantum parameters confirm with the experimental data in all methods of calculation (AM1, PM3 and HF) in the order, except the HOMO in AM1 method. The inhibition efficiency of benzenthiol derivatives obtained Quantum chemically increase with the increase in HOMO energy, and decrease in energy gap $(\Delta \mathrm{E})$. The 4-iso has the highest inhibition efficiency because it had the highest HOMO energy. The inhibition efficiency order did not depend on LUMO energy level. The parameters like hardness $(\eta)$, electro potential chemistry $(\mu)$ and global electrophilicity $(\omega)$ confirms the inhibition efficiency is in the order.

\section{Conflict of interest statement}

Authors declare that they have no conflict of interest.

\section{References}

Al-Kharafi, F.M., 1988. 2-amino-thiazole and 2-amino-4,6dimethylpyrimidine as corrosion inhibitors for copper. Corros. Sci. 28, 163-171.
Al-Mayouf, A.M., Al-Suhybani, A.A., Al-Ameery, A.K., 1998. Corrosion inhibition of $304 \mathrm{SS}$ in sulfuric acid solutions by 2 -methyl benzoazole derivatives. Desalin. 116, 25-33.

Antonijevic, M., Petrovic, M. B., 2008. Copper corrosion inhibitors. A review. Int. J. Electrochem. Sci. 3, 1-28.

Bereket, G., Ogretir, C., Yurt, A., 2001. Quantum mechanical calculations on some 4-methyl-5-substituted imidazole derivatives as acidic corrosion inhibitor for zinc. J. Mol. Struct. (Theochem) 571, 139-145.

Domenicano, A., Hargittai, I., 1992. Accurate Molecular Structures, Their Determination and Importance. Oxford University Press, New York.

Ebenso, E.E., Eddy, N.O., Odongenyi, A.O., 2008. Corrosion inhibitive properties and adsorption behaviour of ethanol extract of Piper guinensis as a green corrosion inhibitor for mild steel in H2S04 . Afr. J. Pure Appl. Chem. 4(11), 107-115.

El-Ashry, H.E., El Nemr, A., Esawy, S.A., Ragab, S., El Ashry, H. E., El Nemr, A., Esawy, S. A., Ragab, S., 2006. Corrosion inhibitors. Part II: Quantum chemical studies on the corrosion inhibitions of steel in acidic medium by some triazole oxadiazole and thiadiazole derivatives. Electrochem. Acta. 51, 3957-3968.

Elmorsi, M.A., Hassanein, A.M., 1999. Corrosion inhibition of copper by heterocyclic compounds. Corros. Sci. 41, 23372352.

Growcock, F.B., 1989. Inhibition of steel corrosion in $\mathrm{HCl}$ by derivatives of cinnamaldehyde: Part I. Corrosion inhibition model. Corrosion. 45, 1003-1007.

Ju, H., Kai, Z.P., Li, Y., 2008. Aminic nitrogen-bearing polydentate Schiff base compounds as corrosion inhibitors for iron in acidic media: a quantum chemical calculation. Corros. Sci. 50, 865-871.

Kendig, M., Jeanjaquet, S., 2002. Cr(VI) and Ce(III) inhibition of oxygen reduction on copper. J. Electrochem. Soc. 149, B47-B51.

Lukovits, I., Kalman, E., Zucchi, F., Corrosion inhibitorscorrelation between electronic structure and efficiency. Corrosion. 57, 3-8.

Ma, H., Chen, S., Niu, L., Zhao, S., Li, S., Li, D., 2002. Inhibition of copper corrosion by several Schiff bases in aerated halide solutions. J. Appl. Electrochem. 32(1), 65-72.

Ma, H.Y., Yang, C., Yin, B.S., Li, G.Y., Chen, S.H., Luo, J.L., 2003. Electrochemical characterization of copper surface modified by n-alkanethiols in chloride-containing solutions. J. Appl. Surf. Sci. 218, 144-154.

Noor, E.A., 2008. Comparative study on the corrosion inhibition of mild steel by aqueous extract of fenugreek seeds and leaves in acidic solutions. J. Eng. Appl. Sci. 3(1), 23-30.

Obi-Egbedi, N.O., Obot, I.B., El-Khaiary, M.I., Umoren, S.A., Ebenso, E.E., 2011. Computational simulation and statistical analysis on the relationship between corrosion inhibition efficiency and molecular structure of some phenanthroline derivatives on mild steel surface. Int. J. Electro Chem. Sci. 6, 5649-5675. 
Obot, I.B., Obi-Egbedi, N.O., 2008a. Inhibitory effect and adsorption characteristics of 2, 3-diaminonaphthalene at aluminum/hydrochloric acid interface: experimental and theoretical study. Surface Rev. Lett. 15(6), 903-910.

Obot, I.B., Obi-Egbedi, N.O., 2008b. Fluconazole as an inhibitor for aluminium corrosion in $0.1 \mathrm{M} \mathrm{HCl}$. Colloids and surfaces A: Physicochem. Eng. Aspects. 330, 207212.

Obot, I.B., Obi-Egbedi, N.O., Umoren, S.A., 2009. The synergistic inhibitive effect and some quantum chemical parameters of 2, 3-diaminonaphthalene and iodide ions on the hydrochloric acid corrosion of aluminium. Corros. Sci. 52, 276-282.

Obot, I.B., Obi-Egbedi, N.O., Umoren, S.A., 2009. Adsorption characteristics and corrosion inhibitive properties of clotrimazole for aluminium corrosion in hydrochloric acid. Int. J. Electrochem. Sci. 4, 863-877.

Oguzie, E.E., 2006. Adsorption and corrosion inhibitive properties of Azadirachta indica in acid solutions. Pigment Resin Technol. 35(6), 334-340.

Otmacic, H., Telegdi, J., Papp, K., Stupnisek-Usac, E., 2004. Protective Properties of an inhibitor layer formed on copper in neutral chloride solution. J. Appl. Electrochem. 34(5), 545-550.
Quraishi, M. A., Wajid Khan, M. A., Ajmal, A., Muralidharan, S., 1996. Influence of substituted benzothiazoles on corrosion in acid solution. J. Appl. Electrochem. 26, 12531258.

Quraishi, M.A., Khan, M.A., Wajid, M.A., Ajmal, M., Muralidharan, S., Iyer, S., Venkatakrishna, S., 1997. Influence of molecular structure of substituted benzothiazoles on corrosion inhibition and hydrogen permeation through mild steel in sulphuric acid. Br. Corros. J. 32(1), 72-76.

Sankarapapavinasam, S., Ahmed, M.F., 1992. Benzenethiols as inhibitors for the corrosion of copper. J. Appl. Electrochem. 22 (4), 390-395.

Scendo, M., Poddebniak, D., Malyszko, J., 2003. Indole and 5chloroindole as inhibitors of anodic dissolution and cathodic deposition of copper in acidic chloride solutions. J. Appl. Electrochem. 33, 287-293.

Wang, C., Chen, S., Zhao, S., 2004. Inhibition effect of ACtreated, mixed self-assembled film of phenylthiourea and 1-dodecanethiol on copper corrosion. J. Electrochem. Soc. 151, B11-B15.

\section{How to cite this article:}

Al-Baalawi, M. T. K., Muhammed, M.K., 2016. Theoretical evaluation of corrosion inhibition performance of some benzenthiol derivatives for copper in acid media. Int. J. Curr. Res. Biosci. Plant Biol. 3(1), 53-57. doi: http://dx.doi.org/10.20546/ijcrbp.2016.301.006 